\title{
Stationäre Zustände in der relativistischen Quantentheorie der Wellenfelder
}

\author{
Von W. Heisenberg \\ Aus dem Max-Planck-Institut für Physik, Göttingen \\ (Z. Naturforschg. 5 a, 367-373 [1950]; eingegangen am 26. Mai 1950)
}

\begin{abstract}
Da in einer konvergenten relativistischen Quantentheorie einstweilen nur die S-Matrix des Systems als ein sicherer Bestandteil des mathematischen Formalismus betrachtet werden kann, wird im ersten Teil der Arbeit auseinandergesetzt, wie man aus dieser Matrix die stationären Zustände freier Teilchen oder zusammengesetzter Systeme ermitteln kann. Die Ergebnisse werden auf den speziellen Ansatz $S=T\left(T^{*} T\right)^{-1 / 2}$ angewendet, der in einer früheren Arbeit angegeben worden war. Dabei werden die besonderen Eigenschaften dieses Ansatzes, wie Invarianz und Auswahlregeln, studiert und gezeigt, daß der Formalismus für Vorgänge mit kleiner Energieübertragung in den alten Hamiltonschen Formalismus übergeht (Korrespondenz). Schließlich wird die Störungstheorie des Formalismus bis zur 2. Näherung durchgeführt.
\end{abstract}

$\mathrm{I}_{\mathrm{f}}^{\mathrm{n}}$ der relativistischen Quantentheorie der Wellenfelder geht man zweckmäßig von der sogenannten Wechselwirkungsdarstellung aus, in der man eine relativistisch invariante Vertauschungsrelation der Wellenfunktionen vorgibt. Die Wellenfunktionen genügen dann einer mit dieser V.R. eindeutig verknüpften linearen Wellengleichung.

Man kann weiter eine Wechselwirkungsenergiedichte $H(x)$, die sich durch die Wellenfunktionen ausdrücken läßt, einführen und aus ihr, wenn das ganze Verfahren konvergiert, nach der Dy s on schen Formel

$$
\begin{aligned}
S= & \sum_{0}^{\infty} \frac{(-i)^{n}}{n !} \\
& \cdot \int_{-\infty}^{+\infty} d x_{1} \ldots \int_{-\infty}^{+\infty} d x_{n} P\left(H\left(x_{1}\right), H\left(x_{2}\right) \ldots H\left(x_{n}\right)\right)
\end{aligned}
$$

die S-Matrix des Systems berechnen ( $P$ bedeutet das Produkt der $H(x)$ in einer solchen Reihenfolge, daß spätere $x$-Werte stets links von früheren $x$-Werten stehen).

Die Frage, ob sich in dieser Weise eine geschlossene Theorie entwickeln läßt, ist in einer vorangehenden Arbeit des Verf. ${ }^{1}$ unter der Voraussetzung untersucht worden, daß man von einer regulären V.R. ausgeht und die Gl. (1) durch eine etwas allgemeinere ersetzt, die der Tatsache Rechnung trägt, daß bei einer regulären V.R. der Ausdruck für $H(x)$ im all-

$1 \mathrm{~W}$. H e is e n berg, Zur Quantentheorie der Elementarteilchen, Z. Naturforschg. 5a, 251 [1950]. Im folgenden stets als I zitiert. Dort finden sich auch die wichtigsten Literaturangaben zum Problem der Arbeit. gemeinen nicht hermitisch sein wird. Diese Frage der Konvergenz und Verallgemeinerung von (1) soll aber erst im 2. Abschnitt behandelt werden.

\section{Berechnung der stationären Zustände aus der S-Matrix}

Im folgenden soll es sich um die Berechnung stationärer Zustände in dem geschilderten Formalismus handeln; z. B. etwa in der Quantenelektrodynamik um die Berechnung eines Zustandes, in dem ein Elektron und ein Positron ähnlich wie im Wasserstoffatom aneinander gebunden sind. Dabei soll aber nicht in der üblichen Weise von der Hamilton-Funktion, sondern um der späteren Verallgemeinerung willen von der S-Matrix ausgegangen werden.

Der Hilbert-Vektor $\Psi$ eines Zustandes bezieht sich in der Wechselwirkungsdarstellung bekanntlich im allgemeinen auf eine dreidimensionale „Fläche“ $\sigma$, die die Eigenschaft haben muß, daß beliebige Punkte in ihr stets durch raumartige Vektoren verbunden sind. Wir betrachten nun nicht beliebige Flächen $\sigma$, sondern wählen eine einparametrige Schar solcher Flächen aus, indem wir von einer willkürlichen Grundfläche $\sigma_{0}$ ausgehen und alle anderen Flächen dadurch erhalten, daß wir die Fläche $\sigma_{0}$ um den Vektor $\varepsilon_{\mu} \tau$ verschieben, wobei $\varepsilon_{\mu}$ ein Einheitsvektor mit beliebiger zeitartiger Richtung und $\tau$ der Parameter der Verschiebung ist. Der Hilbert-Vektor soll zunächst nur für Flächen dieser Schar bestimmt werden, er ist also dann eine Funktion des Parameters $\tau$ (und der Grundfläche $\sigma_{0}$ ).

Den Energie-Impulsvektor des ungestörten Systems (d. h. der freien Teilchen ohne Wechselwirkung) be- 
zeichnen wir mit $J_{\mu}{ }^{0}$. Wendet man diese Operatoren $J_{\mu}{ }^{0}$ an auf einen Hilbert-Vektor $\Psi_{k}$, der den Zustand

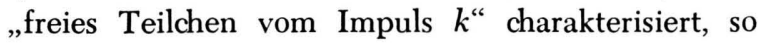
gilt also:

$$
J_{\mu}{ }^{0} \Psi_{k}=k_{\mu} \Psi_{k} \text {. }
$$

Man kann dann von der Wechselwirkungsdarstellung zu einer etwas verallgemeinerten SchrödingerDarstellung übergehen, indem man

$$
X=e^{i J_{\mu^{0}} \varepsilon_{\mu} \tau} \Psi^{\prime}
$$

setzt. Dieser Hilbert-Vektor wird in einem stationären Zustande nur noch in $\operatorname{der}$ Form $e^{i J_{\mu}{ }^{\prime} \varepsilon_{n} \tau}$ von $\tau$ abhängen, wobei $J_{\mu}{ }^{\prime}$ den Energie-Impulsvektor des betreffenden stationären Zustandes bedeutet. Die $J_{i,}$ sind also keine Operatoren, sondern die Eigenwerte von Gesamtenergie und -impuls zu diesem Zustand. In der zeitunabhängigen Darstellung lautet also schließlich der Hilbert-Vektor:

oder

$$
\Phi=e^{i\left(J_{\mu^{0}}-J_{u^{\prime}}\right) \varepsilon_{\mu^{\tau}}} \Psi
$$

$$
\Psi=e^{i\left(J_{\mu^{\prime}}-J_{\mu^{0}}\right)^{0} \varepsilon_{\mu^{\tau}}} \Phi .
$$

In einem stationären Zustande ist $\Phi$ von $\tau$ unabhängig.

Die S-Matrix transformiert ihrer Definition nach den Hilbert-Vektor $\Psi(\tau=-\infty)$ in $\Psi(\tau=+\infty)$. Erstreckt man die Integrale in (1) zunächst nicht von $-\infty$ bis $+\infty$, sondern von einer Fläche mit dem sehr großen negativen Wert $\tau_{1}$ zu einer Fläche mit dem sehr großen positiven Wert $\tau_{2}$, so transformiert diese Matrix $S_{\tau_{1}}^{\tau_{2}}$ den Hilbert-Vektor $\Psi\left(\tau_{1}\right)$ in $\Psi\left(\tau_{2}\right)$. Geht man zur $\Phi$-Darstellung über, so setzt man

$$
U_{\tau_{1}}^{\tau_{2}}=e^{i\left(J_{\mu}{ }^{0}-J_{\mu}{ }^{\prime}\right) \varepsilon_{\mu} \tau_{2}} S_{\tau_{1}}^{\tau_{2}} e^{-i\left(J_{\mu}{ }^{0}-J_{\mu^{\prime}}\right) \varepsilon_{\mu} \tau_{1}}
$$

und die Matrix $U_{\tau_{1}}^{\tau_{2}}$ transformiert $\Phi\left(\tau_{1}\right)$ in $\Phi\left(\tau_{2}\right)$.

Man kann nun die stationären Zustände durch die Aussage charakterisieren, daß der Ausdruck $U_{\tau_{1}}^{\tau_{2}} \Phi\left(\tau_{1}\right)$ von $\tau_{2}$ unabhängig sein soll. Dabei wird es im Sinne der S-Matrix-Theorie genügen, dies für hinreichend große negative Werte von $\tau_{\mathbf{1}}$ zu fordern, $d . h$. zu verlangen, daß $U_{\tau_{1}}^{\tau_{2}} \Phi\left(\tau_{1}\right)$ für $\tau_{1} \rightarrow-\infty$ einem Grenzwert zustrebt. Die Eigenwerte $J_{\mu}{ }^{\prime}$ der stationären Zustände sind also diejenigen Werte $J_{\mu}{ }^{\prime}$, für die $U_{\tau_{1}}^{\tau_{2}} \Phi\left(\tau_{1}\right)$ im limes $\tau_{1} \rightarrow-\infty$ einem von $\tau_{2}$ unabhängigen Grenzwert zustrebt. Für die Bestimmung der stationären Zustände genügt es also nicht ganz, $S_{-\infty}^{+\infty}$ bzw. $U_{-\infty}^{+\infty}$ zu kennen, man muß vielmehr auch noch wissen, daß $U_{\tau_{1}}^{\tau_{2}} \Phi\left(\tau_{1}\right)$ für $\tau_{1} \rightarrow-\infty$ einem Grenzwert zustrebt ${ }^{2}$.

Es soll nun der Zusammenhang dieser Aussage mit dem üblichen Hamilton-Formalismus hergestellt werden. $\mathrm{Zu}$ diesem Zweck schreiben wir die genannte Bedingung in der Form

$$
\frac{d}{d \tau_{2}} U_{\tau_{1}}^{\tau_{2}} \Phi\left(\tau_{1}\right)=0 \text { für } \tau_{1} \rightarrow-\infty .
$$

Es folgt

$$
\left\{i\left(J_{\mu}{ }^{0}-J_{\mu}{ }^{\prime}\right) \varepsilon_{\mu}+e^{i J_{\mu^{0} \varepsilon_{\mu} \tau_{2}}} i \int_{\sigma_{\tau_{2}}} d \sigma_{\mu} \varepsilon_{\mu} H(x) e^{-i J_{\mu}{ }^{0} \varepsilon_{\mu} \tau_{2}}\right\} U_{\tau_{1}}^{\tau_{2}} \Phi\left(\tau_{1}\right)=0 .
$$

( $d \sigma_{\mu}$ bezeichnet einen Vektor, der an der Stelle des Flächenelements $d \sigma$ senkrecht auf der Fläche $\sigma$ steht).

Der Operator in der Klammer ist von $\tau_{2}$ unabhängig, da die Faktoren $e^{i J_{\mu} \varepsilon_{\mu} \tau_{2}}$ und $e^{-i J_{\mu} \varepsilon_{\mu} \tau_{2}}$ einfach die Fläche $\sigma_{\tau_{2}}$ in $\sigma_{0}$ zurückverschieben. Statt (7) kann man also schreiben

$$
\left\{\left(J_{\mu}{ }^{0}-J_{\mu}{ }^{\prime}\right) \varepsilon_{\mu}+\int_{\sigma_{0}} d \sigma_{\mu} \varepsilon_{\mu} H(x)\right\} \Phi\left(\tau_{2}\right)=0 .
$$

Diese Gleichung ist tatsächlich identisch mit der üblichen Hamilton-Gleichung, in der nur Operatoren vorkommen, die von $\tau_{2}$ unabhängig sind. Dabei wird die Fläche $\sigma_{0}$ gewöhnlich als eine zum Vektor $\varepsilon_{u}$ senkrechte ebene Fläche angenommen. Diese Annahme ist aber nicht notwendig, denn die Lösungen der Gl. (8) für verschiedene Flächen $\sigma_{0}$ gehen durch einfache kanonische Transformationen auseinander hervor, wie weiter unten besprochen wird. Insbesondere können also die Eigenwerte $J_{\mu}{ }^{\prime}$ nicht von der Wahl von $\sigma_{0}$ abhängen.

Bevor Gl. (8) auf die Fälle erweitert wird, in denen $S$ nicht in der einfachen Form (1) dargestellt werden

2 Diese Feststellung hängt eng zusammen mit einem Ergebnis von K. Wildermuth (Z. Physik 127, 85 [1949]), daß es bei der Berechnung der stationären Zustände aus den Eigenwerten der S-Matrix, d. h. aus den Phasenfaktoren für Streuung (und zwar nach $\mathrm{Kramers}$ aus den Polen dieser Faktoren auf der imaginären $k$ Achse) nicht nur auf das Verhalten der Phasenfaktoren auf der imaginären $k$-Achse, sondern auch auf das Verhalten in einer infinitesimalen Umgebung dieser Achse ankommt. 
kann, soll noch die allgemeine Form der Eigenfunktionen, d. h. der Hilbert-Vektoren eines stationären Zustandes besprochen werden. Dabei wird es zweckmäßig sein, den Zustandsvektor irgendeines stationären Zustandes durch den Zustandsvektor $\Psi_{0}=\Phi_{0}$ des Vakuums auszudrücken. Als Operatoren, die auf $\Phi_{0}$ wirken, braucht man dabei die Wellenfunktionen $\psi(x)$ oder im Raum der Wellenzahlen $\psi(k)$. Ferner sei $\psi^{-}(k)$ in der Schwingerschen Bezeichnungsweise ein Operator, der ein Teilchen vom Impuls $k$ erzeugt [dabei enthält $\psi(k)$ einen Faktor $\delta\left(k_{v}{ }^{2}+\varkappa^{2}\right)$, wo $\varkappa$ die Ruhmasse eines freien Teilchens ist]. Der HilbertVektor eines stationären Zustandes, bei dem zwei Teilchen aneinander gebunden sind, wird sich dann, in Analogie zu den Verhältnissen in der unrelativistischen Theorie, darstellen lassen in der Form

oder

$$
\begin{gathered}
\Psi(\sigma)=\int_{\sigma} d \sigma_{\lambda} J_{\lambda^{\prime}} e^{i\left(J_{\mu}-k_{\mu}-k_{\mu}{ }^{\prime}\right) x_{\mu}} \psi^{-}(k) \psi^{-}\left(k^{\prime}\right) \chi\left(k, k^{\prime}\right) d k d k^{\prime} \Psi_{0} \\
\Phi=\int_{\sigma_{0}} d \sigma_{\lambda} J_{\lambda^{\prime}} e^{i\left(J_{\mu}-k-k^{\prime}\right) x_{\mu}} \psi^{-}(k) \psi^{-}\left(k^{\prime}\right) \chi\left(k, k^{\prime}\right) d k d k^{\prime} \Psi_{0} .
\end{gathered}
$$

Der Parameter $\tau$ kommt in $\Phi$ nicht mehr vor; $\Phi$ muß aber, um einen stationären Zustand darzustellen, der Gl. (8) genügen, die ja auch von $\tau$ unabhängig ist. $\chi\left(k, k^{\prime}\right)$ ist irgendeine für den Zustand charakteristische Funktion der Vektoren $k$ und $k^{\prime}$. Natürlich ${ }^{\circ}$ wird es im allgemeinen gar keine stationären $\mathrm{Zu}$ stände geben, bei denen die „Teilchenzahl“ genau festgelegt ist; Gl. (9) sollte aber nur an einem speziellen Fall die Struktur der Eigenfunktionen zeigen. Ganz allgemein wird man die Eigenfunktion eines stationären Zustandes schreiben können:

$$
\Psi(\sigma)=\int_{\sigma} d \sigma_{\lambda} J_{\lambda^{\prime}} e^{i\left(J_{\mu}{ }^{\prime}-J_{\mu}{ }^{0}\right) x_{\mu}} O\left[\psi(k), \psi\left(k^{\prime}\right) \ldots\right] \Psi_{0},
$$

wobei $O\left[\psi(k), \psi\left(k^{\prime}\right)\right]$ ein Operator ist, der irgendwie von den Wellenfunktionen in der Impulsraumdarstellung abhängt. $\Phi$ erhält man wieder aus $\Psi(\sigma)$, indem man $\sigma$ durch $\sigma_{0}$ ersetzt.

Es ist noch zu zeigen, daß die Gl. (9) bzw. (10) für jede beliebige Fläche $\sigma_{0}$ eine Lösung von (8) darstellt $\left[\sigma_{0}\right.$ ist dabei stets die Fläche, die auch in der Schrödinger-Gleichung (8) vorkommt], wenn dies für ein bestimmtes $\sigma_{0}$ zutrifft. Um dies einzusehen, kann man sich zunächst überlegen, daß der auf $\Psi_{0}$ wirkende Ausdruck in (9) bzw. (10) unter dem Integralzeichen an der Stelle $x$ nur von den Wellenfunktionen an der Stelle $x$ oder von ihren Werten im Zukunfts- oder Vergangenheitskegel von $x$ abhängt. Daraus folgt, daß $H\left(x^{\prime}\right)$ mit diesem Ausdruck überall vertauschbar ist, außer an der Stelle $x=x^{\prime}$. Da $\Psi_{0}$ der HilbertVektor des Vakuums ist, wirkt der Operator $H\left(x^{\prime}\right)$ also nur auf den Teil des Hilbert-Vektors in einer vom Vakuumfall verschiedenen Weise, der zu $x=x^{\prime}$ gehört. Für diesen Teil aber ergänzt sich der Faktor $e^{i\left(J_{\mu}{ }^{0 \prime \prime}-J_{\mu}{ }^{0 \prime}\right) x_{\mu}}$, der $\mathrm{zu}$ einem Matrixelement $\left(J_{\mu}{ }^{0^{\prime}}|H(x)| J_{\mu}{ }^{0^{\prime \prime}}\right)$ gehört, mit dem Faktor $e^{i\left(J_{\mu}{ }^{\prime}-J_{\mu}{ }^{0 \prime \prime}\right) x_{\mu}}$ [des Matrixelements $\left(J_{\mu}{ }^{0^{\prime \prime}}|O|\right)$ ] zu dem Faktor $e^{i\left(J_{\mu^{\prime}}-J_{\mu^{0}}{ }^{\prime \prime}\right) x_{\mu}}$, der nun für alle Matrixelemente der Gleichung den gleichen Wert hat und daher als gemeinsamer Faktor vorgezogen werden kann. Damit ist die Unabhängigkeit von $\sigma_{0}$ bewiesen. Der von der Form von $\sigma$ oder $\sigma_{0}$ unabhängige Kern der Eigenfunktion ist also der Operator $O\left(\psi^{(k)}, \ldots\right)$, der dann und nur dann einen stationären Zustand beschreibt, wenn (9) bzw. (10) eine Lösung von (8) ist. In diesem Operator kommen die Raum-Zeit-Koordinaten überhaupt nicht mehr vor.

2. Erweiterung für den Fall nichthermitischer Ausdrückefür $H(x)$

Wenn man von einer regulären V.R. ausgeht, so kann die Wellenfunktion $\bar{\psi}$, die mit $\psi$ in der regulären V.R. steht, nicht hermitisch zugeordnet zu $\psi$ sein (vgl. l.c.I). Denn wenn man die reguläre V.R. in der Form schreibt

$$
S^{R}\left(x-x^{\prime}\right)=\sum c_{l} S_{l}\left(x-x^{\prime}\right),
$$

wobei die $S_{l}\left(x-x^{\prime}\right)$ die üblichen singulären V.R. für die Teilchen der Masse $\varkappa_{l}$ bedeuten, so müssen einige $c_{l}$ negativ sein. Daraus folgt, daß man dann definieren muß:

$\psi=\sum_{\mathrm{c}_{l}>0} \alpha_{l} \psi_{l}+\underset{\mathrm{c}_{l}<0}{j \sum_{l} \beta_{l}} \psi_{l} ; \bar{\psi}=\sum_{\mathrm{c}_{l}>0} \bar{\alpha}_{l} \bar{\psi}_{l}+j \sum_{\mathrm{c}_{l}<0} \bar{\beta}_{l} \bar{\psi}_{l}$, mit $\left|\alpha_{l}^{2}\right|=c_{l}$ bzw. $\left|\beta_{l}^{2}\right|=-c_{l}$ und $j^{2}=-1$.

Die $\bar{\psi}_{l}$ sind die im Sinne der Diracschen Theorie zu $\psi_{l}$ hermitisch zugeordneten Größen. (Sie gehen aus $\psi_{l}$ durch Umkehrung der quantentheoretischen $\sqrt{-1}$ in $i \hbar$ hervor, ohne Umkehrung der relativistischen $\sqrt{-1}$ in $i c t$; kehrt man beide imaginäre Einheiten 
gleichzeitig um, so erhält man bekanntlich die Größe $\psi_{l}^{*}$, die sich von $\bar{\psi}_{l}$ um den Faktor $\gamma_{4}$ unterscheidet.) Neben den beiden ebengenannten imaginären Einheiten gibt es also jetzt auch noch die Größe $i$, die gewissermaßen in ähnlicher Weise zur „kleinsten Länge" gehört, wie die anderen beiden zu $\hbar$ bzw. $c$ gehören.

Eine aus $\bar{\psi}$ und $\psi$ in der üblichen Weise zusammengesetzte Wechselwirkungsfunktion $H(x)$ ist daher zwar gegenüber der Vorzeichenumkehr der ersten beiden imaginären Einheiten, nicht aber gegenüber der Umkehr von $j$ invariant. Es wurde daher in I vorgeschlagen, zunächst eine Matrix $T$ nach der Formel

$T=\sum_{0}^{\infty} \frac{(-i)^{n}}{n !} \int_{-\infty}^{+\infty} d x_{1} \ldots \int_{-\infty}^{+\infty} d x_{n} P\left(H\left(x_{1}\right), \ldots H\left(x_{n}\right)\right)$

zu bilden und dann

$$
S=T\left(T^{*} T\right)^{-1 / 2}
$$

zu setzen. Die Matrix $S$ ist im Gegensatz zu $T$ unitär, wie es sein muß.

Außerdem ist $S$ gegen eine Vorzeichenumkehr von $j$ invariant. Dies kann man in der folgenden Weise einsehen: Aus (13) folgt zunächst

$$
T_{j}^{*}=T_{-j}^{-1} .
$$

Dann findet man durch Umformung:

$$
\begin{aligned}
S_{j} & =T_{j}\left(T_{j}^{*} T_{j}\right)^{-1 / 2}=T_{j}\left(T_{-j}^{-1} T_{-j}^{*}\right)^{-1 / 2} \\
& =T_{j}\left(T_{-j}^{*} T_{-j}\right)^{1 / 2}=T_{j} T_{-j}^{*} T_{-j}\left(T_{-j}^{*} T_{-j}\right)^{-1 / 2} \\
& =T_{-j}\left(T_{-j}^{*} T_{-j}\right)^{-1 / 2}=S_{-j} .
\end{aligned}
$$

Die damit bewiesene Invarianz von $S_{j}$ gegenüber Vorzeichenumkehr von $i$ bedeutet physikalisch, daß die Matrix $S_{j}$ keinerlei Übergänge vermittelt, bei denen eine ungerade Anzahl von Teilchen mit positivem $c_{l}$ sich in eine ungerade Anzahl von Teilchen mit negativem $c_{l}$ verwandelt. Für die beiden Teilchengruppen (mit positivem bzw. negativem $c_{l}$ ) gelten also streng zwei unabhängige Erhaltungssätze der „Ladung“. Tatsächlich gibt es ja neben dem gewöhnlichen Erhaltungssatz der Ladung noch den Erhaltungssatz der Nukleonenzahl, der z. B. verhindert, daß ein Proton sich etwa in ein Positron und ein oder mehrere Lichtquanten verwandelt. Doch soll auf die Anwendung der mathematischen Ergebnisse auf die empirischen Elementarteilchen hier nicht weiter eingegangen werden.

Wir wiederholen nun die Überlegungen des ersten Abschnitts für die Matrix in (14) und definieren

und

$$
V_{\tau_{1}}^{\tau_{2}}=e^{i\left(J_{\mu}{ }^{0}-J_{\mu}{ }^{\prime}\right) \tau_{2}} T_{\tau_{1}}^{\tau_{2}} e^{-i\left(J_{\mu}{ }^{0}-J_{\mu}{ }^{\prime}\right) \tau_{1}}
$$

$$
U_{\tau_{1}}^{\tau_{2}}=V_{\tau_{1}}^{\tau_{2}}\left(V_{\tau_{1}}^{\tau_{2}^{*}} V_{\tau_{1}}^{\tau_{2}}\right)^{-1 / 2}
$$

Dann gilt zunächst

$$
\frac{d}{d \tau_{2}} V_{\imath_{1}}^{\tau_{2}}=i\left\{\left(J_{\mu}^{0}-J_{\mu^{\prime}}\right) \varepsilon_{\mu}+\int_{\sigma_{0}} d \sigma_{\mu} \varepsilon_{\mu} H(x)\right\} V_{\tau_{1}}^{\tau_{2}}
$$

und

$$
\frac{d}{d \tau_{2}}\left(V_{\tau_{1}}^{\tau_{2}^{*}} V_{\tau_{1}}^{\tau_{2}}\right)=i V_{\tau_{1}}^{\tau_{2}^{*}} \int_{\sigma_{0}} d \sigma_{\mu} \varepsilon \mu\left(H(x)-H^{*}(x)\right) V_{\tau_{1}}^{\tau_{2}}
$$

Definiert man weiter

$$
\frac{d}{d \tau_{2}}\left(V_{\tau_{1}}^{\tau_{2 *}} V_{\tau_{1}}^{\tau_{2}}\right)^{-1 / 2}=i Z\left(V_{\tau_{1}}^{\tau_{2 *}} V_{\tau_{1}}^{\tau_{2}}\right)^{-1 / 2},
$$

so wird

$$
Z=\lim _{\delta \rightarrow 0} \frac{1}{\delta}\left\{\left(V_{\tau_{1}}^{\tau_{2} *}\left[1+\delta \int_{\sigma_{0}} d \sigma_{\mu} \varepsilon_{\mu}\left(H-H^{*}\right)\right] V_{\tau_{1}}^{\tau_{2}}\right)^{-1 / 2}\left(V_{\tau_{1}}^{\tau_{2}^{*}} V_{\tau_{1}}^{\tau_{2}}\right)^{1 / 2}-1\right\},
$$

was wegen der Nichtvertauschbarkeit der auftretenden Größen zunächst nicht weiter vereinfacht werden kann.

Aus der Forderung, daß $U_{\tau_{1}}^{\tau_{2}} \Phi\left(\tau_{1}\right)$ einem von $\tau_{2}$ unabhängigen Grenzwert für $\tau_{1} \rightarrow-\infty$ zustreben soll, folgt dann in Analogie zu (8)

$$
\left\{\left(J_{\mu}^{0}-J_{\mu}{ }^{\prime}\right) \varepsilon_{\mu}+\int_{\sigma_{0}} d \sigma_{\mu} \varepsilon_{\mu} H(x)+V Z V^{-1}\right\} \Phi\left(\tau_{2}\right)=0,
$$

wobei statt $V_{-\infty}^{\tau_{2}}$ einfach $V$ geschrieben ist. Da $S$ und $U$ gegen Vorzeichenänderung von $j$ invariant sind, kann man zu (23) auch dieselbe Gleichung mit entgegengesetztem $i$ addieren und erhält als symmetrische Form der Schrödinger-Gleichung

$$
\left\{\left(J_{\mu}^{0}-J_{\mu}{ }^{\prime}\right) \varepsilon_{\mu}+\int_{\sigma_{0}} d \sigma_{\mu} \varepsilon_{\mu} \frac{1}{2}\left[H+H^{*}\right]+\frac{1}{2}\left(V_{j} Z_{j} V_{i}^{-1}+V_{-j} Z_{-j} V_{-j}^{-1}\right)\right\} \Phi=0 .
$$


Neben den Gliedern mit der Wechselwirkung $1 / 2\left[H(x)+H^{*}(x)\right]$ enthält die Gleichung noch die Ausdrücke mit $V_{-\infty}^{\tau_{2}}$, die für die Abweichung vom Hamilton-Formalismus charakteristisch sind. Zunächst kann gezeigt werden, daß diese Glieder entgegen dem äußeren Anschein nicht von $\tau_{2}$ abhängen. Denn die Potenzreihenentwicklung (13) von $T_{\tau_{1}}^{\tau_{2}}$ lehrt, daß jedes Matrixelement $\left(\mathbf{J}^{\prime}|T| \mathbf{J}^{\prime \prime}\right)$ als Zeitfaktor das Integral

$$
\int_{\tau_{1}}^{\tau_{2}} e^{i\left(J_{\mu}{ }^{0 \prime \prime}-J_{\mu}{ }^{0 \prime}\right) \varepsilon_{\mu} \tau} d t
$$

enthält, das für $\tau_{1}=-\infty$ einfach durch

$$
\frac{e^{i\left(J_{\mu}{ }^{0 \prime \prime}-J_{\mu}{ }^{\prime \prime}\right) \varepsilon_{\mu}} \tau_{2}}{i\left(J_{\mu}{ }^{0^{\prime \prime}}-J_{\mu}{ }^{0^{\prime}}\right) \varepsilon_{\mu}}
$$

ersetzt werden kann.

Bei der Bildung der Ausdrücke $V Z V^{-1}$ heben sich die Exponentialfaktoren heraus und es bleibt von den Zeitfaktoren nur noch $1 / i\left(J_{\mu}{ }^{0^{\prime \prime}}-J_{\mu}{ }^{0^{\prime}}\right) \varepsilon_{\mu}$ übrig, was von $\tau_{2}$ unabhängig ist. Dabei erscheint es im ersten Augenblick unplausibel, daß der Operator $V_{-\infty}^{\tau_{2}}$, der ja ein unendlich großes Zeitintervall überbrückt, noch Übergänge enthält, bei denen die Energie sich ändert. Dies liegt daran, daß man zwar unter $V_{-\infty}^{+\infty}$ gewöhnlich den Operator versteht, der sich ergibt, wenn man bei $\tau=-\infty$ die Wechselwirkung adiabatisch einschaltet und sie bei $\tau=+\infty$ wieder adiabatisch ausschaltet; dann enthält der Operator nur Übergänge, bei denen sich $J_{\mu}{ }^{0}$ nicht ändert. Bei $V_{-\infty}^{\tau_{2}}$ aber wird die Wechselwirkung

$\left\{\left(J_{\mu} \mu^{0}-J_{\mu}{ }^{\prime}\right) \varepsilon \mu+\int_{\sigma_{0}} d \sigma_{\mu} \varepsilon \mu \frac{1}{2}\left(H+H^{*}\right)+\frac{i}{8}\left[e^{i J_{\mu}{ }^{0} \varepsilon_{\mu} \tau_{2}}\right.\right.$

(hierbei ist die Abkürzung $[a, b]=a b-b a$ benützt).

Berechnet man aus dieser Gleichung den Eigenwert eines Zustandes in zweiter Näherung, so erkennt man deutlich, in welcher Weise der Formalismus die unendlichen Selbstenergien beseitigt. Setzt man nämlich $H=A+j B$, so würde man vom ersten klassischen Wechselwirkungsglied der Gl. (25) als Zusatz zweiter Ordnung zur Selbstenergie nur den bekannten Ausdruck

$$
\sum_{k} \frac{A_{l k} A_{k l}}{E_{k}-E_{l}}
$$

erhalten, der im allgemeinen divergiert. Dazu kommt aber nach (25) aus den nicht-Hamiltonschen Gliedern ja nicht adiabatisch ausgeschaltet, sie ist zur Zeit $\tau_{2}$ noch in voller Stärke vorhanden. Daher stellt $V_{-\infty}^{\tau_{2}}$ die Transformation dar, die von den Zuständen vor adiabatischer Einschaltung der Wechselwirkung zu denen nach Einschaltung führt; dabei kann es auch Änderungen der $J_{\mu}{ }^{0}$ geben.

Die Operatoren $V$ in (24) können, anders als in der gewöhnlichen Hamilton-Theorie, am Zustandsvektor $\Phi$ raumzeitliche Mittelungen über Gebiete von der Größenordnung der kleinsten Länge ausführen, die das charakteristische Merkmal jeder SMatrix-Theorie sind. Daher kann auch der Schluß, daß der Operator $O$ in der Zustandsfunktion von der Form der Fläche $\sigma_{0}$ unabhängig sei, aus dem ersten Abschnitt nicht ohne weiteres übernommen werden. Vielmehr besteht die Unabhängigkeit vielleicht nur dann, wenn die Flächen in Gebieten von der Größenordnung der kleinsten Länge praktisch eben sind. Wir wollen dies also voraussetzen und annehmen, daß die Krümmungsradien der Fläche stets sehr groß gegen die kleinste Länge sind. Dann kann der Operator $O$ jedenfalls aus (23) bzw. (24) ähnlich wie in (8) unabhängig von $\sigma_{0}$ bestimmt werden.

\section{Störungstheorie}

Die Struktur der Schrödinger-Gleichung (24) läßt sich besonders leicht überblicken, wenn man die Wechselwirkung als klein voraussetzt und nach ihr entwickelt. Bricht man die Entwicklung mit dem zweiten Gliede in $H$ ab, so erhält man durch Ausrechnung der Ausdrücke $V Z V^{-1}$ an Stelle von (24):

$\left.\left.\int_{-\infty}^{\tau_{2}}\left(H-H^{*}\right) d x e^{-i J_{\mu}{ }^{0} \varepsilon_{\mu} \tau_{2}}, \int_{\sigma_{0}} d \sigma_{\mu} \varepsilon_{\mu}\left(H-H^{*}\right)\right]_{-}\right\} \Phi=0$

noch der ebenfalls divergierende Ausdruck

$$
-\sum_{k} \frac{B_{k l} B_{l k}}{E_{k}-E_{l}},
$$

der mit dem ersten zusammen sich auch in der Form schreiben läßt

$$
+\sum_{k} \frac{H_{k l} H_{l k}}{E_{k}-E_{l}}
$$

(Ausdrücke der Form $A_{l k} B_{k l}$ verschwinden!), was wegen der regulären V.R. konvergiert.

Im übrigen ist die Benutzung der SchrödingerGleichung (24) zur Ermittlung der Eigenwerte $J_{\mu}{ }^{\prime}$ 
für die Zustände freier Teilchen, die durch die Wechselwirkung modifiziert werden, nicht besonders zweckmäßig. Es ist viel einfacher, den Ausdruck $U_{-\infty}^{\tau_{2}} \Phi_{0}$ direkt durch Reihenentwicklung nach $H(x)$ zu berechnen, wobei $\Phi_{0}$ den ungestörten Zustandsvektor etwa zu einem Zustand mit dem Eigenwert $J_{\mu}{ }^{0^{\prime}}$ bedeutet. Man entnimmt dann $S_{\tau_{1}}^{\tau_{2}}$ aus der Formel (31) der früheren Arbeit und multipliziert von links mit

von rechts mit

$$
e^{i\left(J_{\mu}{ }^{0}-J_{\mu}{ }^{\prime}\right) \varepsilon_{\mu} \tau_{2}},
$$

$$
e^{-i\left(J_{\mu}{ }^{0}-J_{u^{\prime}}\right) \varepsilon_{\mu l} \tau_{1}}
$$

und läßt $\tau_{1}$ später gegen $-\infty$ gehen. Der Eigen- wert $J_{\mu}{ }^{\prime}$ folgt aus der Forderung, daß das Diagonalglied in $U_{\tau_{1}}^{\tau_{2}}$ zu $J_{\mu}{ }^{0}=J_{\mu}{ }^{0^{\prime}}$ von $\tau_{2}$ unabhängig sein muß. Zur Berechnung des Diagonalglieds hat man hier auch

$$
e^{i\left(J_{\mu}^{0 \prime}-J_{\mu}^{\prime}\right) \varepsilon_{\mu}\left(\tau_{2}-\tau_{1}\right)}
$$

nach Potenzen von $\tau_{2}-\tau_{1}$ zu entwickeln, wobei man, wenn man sich möglichst eng an die frühere Störungstheorie anschließen will,

$$
J_{\mu}{ }^{\prime}-J_{\mu^{0 \prime}}=\sum_{n} \Delta J_{\mu}{ }^{(n)}
$$

setzen kann ( $n$ bezeichnet die Potenz von $H$, zu der das betreffende Glied gehört). Nach diesem Verfahren erhält man, wenn man bis zur 2. Näherung geht:

$$
\begin{aligned}
\left(J_{\mu^{0 \prime}}\left|U_{-\infty}^{\tau_{2}}\right| J_{\mu^{0 \prime}}\right)= & \lim _{\tau_{1} \rightarrow-\infty}\left(J_{\mu^{0 \prime}} \mid 1+i \Delta J_{\mu}^{(1)} \varepsilon_{\mu}\left(\tau_{2}-\tau_{1}\right)-\frac{i}{2} \int_{\tau_{1}}^{\tau_{2}}\left(H+H^{*}\right) d x+i \Delta J_{\mu}^{(2)} \varepsilon_{\mu}\left(\tau_{2}-\tau_{1}\right)\right. \\
-\frac{1}{4} \int_{\tau_{1}}^{\tau_{2}} d x_{1} \int_{\tau_{1}}^{\tau_{2}} d x_{2}\left[P\left(H\left(x_{1}\right) H\left(x_{2}\right)\right)\right. & \left.+P\left(H^{*}\left(x_{1}\right) H^{*}\left(x_{2}\right)\right)\right]-\frac{1}{2}\left[\Delta J_{\mu}^{(1)} \varepsilon_{\mu}\left(\tau_{2}-\tau_{1}\right)\right]^{2} \\
& \left.+\frac{1}{2} \Delta J_{\mu}^{(1)} \varepsilon_{\mu}\left(\tau_{2}-\tau_{1}\right) \int_{\tau_{1}}^{\tau_{2}}\left(H+H^{*}\right) d x-\frac{1}{8}\left[\int_{-\infty}^{\tau_{2}} d x\left(H-H^{*}\right)\right]^{2} \mid J_{\mu}{ }^{0 \prime}\right)=\text { const }
\end{aligned}
$$

Durch Vergleich der mit $\tau_{2}-\tau_{1}$ zunehmenden Glieder folgt:

$$
\begin{aligned}
& \Delta J_{\mu}^{(1)} \varepsilon_{\mu}=\lim _{\tau_{1} \rightarrow-\infty} \frac{1}{\tau_{2}-\tau_{1}}\left(J_{\mu}^{0^{\prime}}\left|\frac{1}{2} \int_{\tau_{1}}^{\tau_{2}} d x\left(H+H^{*}\right)\right| J_{\mu}{ }^{0^{\prime \prime}}\right) \\
& \Delta J_{\mu}^{(2)} \varepsilon_{\mu}=\lim _{\tau_{1} \rightarrow-\infty} \frac{-i}{\tau_{2}-\tau_{1}}\left(J_{\mu^{0}}\left|\frac{1}{4} \int_{\tau_{1}}^{\tau_{2}} d x_{1} \int_{-\infty}^{\tau_{2}} d x_{2}\left[P\left(H\left(x_{1}\right) H\left(x_{2}\right)\right)+P\left(H^{*}\left(x_{1}\right) H^{*}\left(x_{2}\right)\right)\right]\right| J_{\mu}{ }^{0^{\prime}}\right) .
\end{aligned}
$$

Die Eigenfunktion des Zustandes $J_{\mu}{ }^{{ }^{\prime}}$, der ein durch die Wechselwirkung modifiziertes freies Teilchen vorstellt, lautet

$$
\Phi=U_{-\infty}^{\tau_{2}} \Phi_{0} .
$$

Wenn ein stationärer Zustand dadurch zustande kommt, daß mehrere Teilchen durch die Wechselwirkung aneinander gebunden sind, so wird allerdings eine so einfache Berechnung des Zustandsvektors kaum möglich sein, vielmehr wird man dann zweckmäßig die Schrödinger-Gleichung (24) benützen.

4. Korrespondenz und Eindeutigkeit des Formalismus

Die Abweichung des vorliegenden Formalismus von der Hamiltonschen Theorie rührt von den imaginären (also zu $i$ proportionalen) Anteilen der Wechselwirkung $H$ her. Dies ist besonders deutlich an Gl. (25) zu sehen.
Die reguläre V.R., die der Theorie zugrunde liegt, enthält als Dimensionskonstante die „kleinste Länge“ $l_{0}$; daher haben die aus der V.R. folgenden Massen der ungestörten Elementarteilchen Werte der Größenordnung $\hbar\left(c l_{0}\right)^{-1}$ (oder in unseren Einheiten $l_{0}^{-1}$ ), auch die Unterschiede der Massen werden von dieser Größenordnung sein.

Die Übergangselemente, die zum imaginären Teil von $H$ gehören und Úbergänge einer ungeraden Zahl von Teilchen mit positivem $c_{l}$ in eine ungerade Zahl von Teilchen mit negativem $c_{l}$ bewirken [vgl. (11) und (12)], sind daher auch, wenn nicht besondere Verhältnisse vorliegen, mit Energieänderungen der Größenordnung $l_{0}^{-1}$ verknüpft.

Betrachtet man nun Vorgänge, bei denen nur viel kleinere Energien $\left(\ll l_{0}^{-1}\right)$ umgesetzt werden, so kann man die Beiträge der imaginären Teile von $H$ in ähnlicher Weise als konstant ansehen, wie man etwa in der Theorie der Bandenspektren die Elektronenenergien als konstant ansehen darf. In diesem 
Fall kehrt man also zu einem reinen Hamilton-Formalismus zurück. Damit ist gezeigt, daß die durch (14) bzw. (24) dargestellte Theorie für kleine Energien $\left(\ll l_{0}^{-1}\right)$ in die bisherige Hamiltonsche Theorie übergeht, während die nicht-Hamiltonschen Glieder bei hohen Energieübertragungen für die Konvergenz des Verfahrens sorgen.

An dieser Stelle entsteht die Frage, ob es neben dem Ausdruck (14) nicht auch noch andere Formeln für $S$ gibt, die ebenso wie (14) $S$ unitär machen und zugleich die Korrespondenz gewährleisten. Man könnte etwa an Formen für $S$ wie $\left(T_{j} T_{-j}\right)^{1 / 2}, T_{j}^{1 / 2} T_{-j}^{1 / 2}$, $T^{1 / 2} T^{*-1 / 2}$ u. dgl. denken. Die Rechnung zeigt aber, daß keiner dieser Ansätze unitär ist. Der einzige Ausdruck, der neben (14) noch in Betracht kommt, lautet

$$
S=\left(T T^{*}\right)^{-1 / 2} T \text {. }
$$

Man kann aber ohne Schwierigkeit sehen, daß er mit dem Ausdruck $T\left(T^{*} T\right)^{-1 / 2}$ identisch ist. Man findet nämlich durch einfache Umformung

$$
\left(T T^{*}\right)^{-1 / 2} T=T\left[T^{-1}\left(T T^{*}\right)^{-1 / 2} T\right]=T\left(T^{*} T\right)^{-1 / 2} .
$$

Daher dürfte die Formel (14) bzw. (29) wohl die einzige einfache Darstellung der S-Matrix durch die $T$-Matrix sein.

\title{
Eine Differentialgleichungstheorie der Elementarteilchen
}

\author{
Von K. Wildermuth \\ Aus dem Max-Planck-Institut für Physik, Göttingen \\ (Z. Naturforschg. 5 a, 373-381 [1950]; eingegangen am 9. Juni 1950)
}

\begin{abstract}
Im ersten Teil wird gezeigt, daß man zur Gewinnung einer divergenzfreien Theorie der Elementarteilchen zwangsläufig vom üblichen Hamilton-Formalismus abweichen muß, falls man nicht ein sehr gekünsteltes Abschneideverfahren für die hohen Impulse 2, durch die in der Quantentheorie der Wellenfelder bekanntlich immer die Divergenzen hervorgerufen werden, einführen will; d. h., falls man nicht den physikalisch sehr einleuchtenden Gedanken aufgeben will, daß durch die Wechselwirkung sämtlicher Teilchen untereinander die Divergenzen der Theorie von selbst verschwinden.

Im zweiten Teil werden ein paar qualitative physikalische Folgerungen besprochen, die man bereits jetzt aus der Theorie entnehmen kann. Wie in der Arbeit von $\mathrm{H}$ e is e $\mathrm{n} \mathrm{b}$ e r g 1 ausgeführt wurde, muß man sich im Rahmen der obigen Theorie die Teilchen mit ganzzahligem Spin als aus Spinorteilchen zusammengesetzt vorstellen. Es zeigt sich nun, daß es auch näherungsweise nicht richtig ist, das Lichtquant als aus Elektron und Positron zusammengesetzt zu betrachten; sondern mit einer gewissen Wahrscheinlichkeit ist es aus Proton und Antiproton usw. zusammengesetzt. Zum Schluß wird dann noch kurz gezeigt, wie man sich eine qualitative Vorstellung über das Zustandekommen des Coulomb-Feldes machen kann.
\end{abstract}

$\mathrm{I}_{\mathrm{m}}^{\mathrm{m}}$ m Anschluß an eine grundlegende Arbeit von $\mathrm{H}$ e is e n berg ${ }^{1}$ werden die dort niedergelegten Gedanken über eine zukünftige Theorie der Elementarteilchen, die bei Heisenberg in die Form einer Integralgleichungstheorie gekleidet sind, mittels einer Differentialgleichungstheorie dargestellt. Diese Darstellung bedeutet bereits eine starke Einschränkung der allgemeinen Heisenbergschen Theorie, da in der letzteren der Grad der Ableitungen begrenzt ist, während eine allgemeine Integralgleichungstheorie äquivalent ist einer Differentialgleichungstheorie unendlich hoher Ordnung. Physikalisch bedeutet das, daß man erstens im Gegensatz zur Heisenbergschen Formulierung das Grundspektrum der Elementarteilchen der wechselwirkungsfreien Theorie diskret und endlich wählen muß. Wie sich nachher zeigen wird, ist nämlich der Grad der höchsten Ableitung, der in der Theorie vorkommt, gleich

1 W. H e is e n b e r g, Z. Naturforschg. 5a, 251 [1950]. der Anzahl (falls man zwischen Teilchen und Antiteilchen unterscheidet, gleich der halben Anzahl) der Elementarteilchen des Grundspektrums. Zweitens werden die Ansätze für die Wechselwirkungsenergie der Teilchen untereinander stark eingeschränkt, da in einer Differentialgleichungstheorie die Wechselwirkungsenergiedichte als gewöhnliche Dichtefunktion darstellbar sein muß. Sie darf also nur von der Wellenfunktion $\psi$ und deren räumlichen und zeitlichen Ableitungen $\partial m \psi / \partial x^{m}$ am betrachteten Raum-Zeitpunkt $x_{v}$, selbst abhängen. Ob die obigen Einschränkungen gerechtfertigt sind, muß sich beim weiteren Ausbau der Theorie zeigen.

Die Differentialgleichungstheorie hat den Vorteil, daß bei ihr die Korrespondenz zur bisherigen Quantenmechanik besonders deutlich wird. So sieht man z. B., daß die Regularisierungsbedingungen für die Vertauschungsrelationen (V.-R.) der Wellenfunktionen untereinander ganz von selbst aus den quantenmechanischen Bewegungsgleichun- 